\title{
Schizophrénie et délétions du chromosome 22q11
}

La schizophrénie est une maladie mentale sévère affectant environ $1 \%$ de la population mondiale. La symptomatologie est caractérisée par une dissociation progressive et chronique de la personnalité, pouvant associer discordance, ambivalence, sentiment de dépersonnalisation, délire, hallucinations, autisme. Son étiologie est inconnue et complexe, et fait vraisemblablement intervenir des facteurs liés à l'environnement et des facteurs génétiques. L'existence d'un déterminisme génétique de la maladie est suggérée par des études familiales et les études de jumeaux [1]. Le risque de développer la maladie est d'environ $12 \%$ pour un individu dont seul un des parents est schizophrène; ce risque passe à $40 \%$ si les deux parents sont atteints. Par ailleurs, des études d'adoption indiquent que les enfants de parents schizophrènes ont un risque de $10 \%$ de développer la maladie, contre $1 \%$ (taux de la population générale) pour les enfants de parents non schizophrènes. Cela montre que l'effet de l'environnement ne peut être tenu pour seul responsable du développement de la maladie. Les études de jumeaux notent que si l'un des jumeaux est schizophrène, le risque pour que le second soit atteint est environ deux ou trois fois supérieur s'il s'agit de vrais jumeaux par rapport à des faux jumeaux.

Le mode de transmission de la schizophrénie n'est pas connu. Les analyses de ségrégation indiquent que la prédisposition à la schizophrénie n'est pas transmise sur un mode mendélien, mais selon un modèle multifactoriel. Des analyses de liaison génétique ont été entreprises pour localiser les gènes de prédisposition.
Cependant, l'analyse de liaison est rendue difficile par l'incertitude concernant le mode héréditaire (dominance, récessivité, oligo- ou polygénicité avec la possibilité d'un gène majeur agissant de concert avec d'autres gènes pour aboutir au déclenchement de la maladie), par une pénétrance incomplète, par l'hétérogénéité génétique probable, et par l'existence de phénocopies*. A cela s'ajoutent les problèmes liés aux difficultés diagnostiques dans le cadre des maladies psychiatriques. Le diagnostic doit être posé de façon rigoureuse en suivant des critères de référence tels que ceux du DSM-III-R [2], afin de définir une population la plus homogène possible de malades pour les analyses de liaison. Les premiers résultats de liaison qui suggéraient la localisation de gènes de prédisposition sur les chromosomes $5 q$ et $11 \mathrm{q}$ ont rapidement été mis en doute par d'autres études, créant ainsi une certaine désillusion vis-à-vis de cette approche pour la schizophrénie (pour revue, voir. [3, 4]). L'utilisation actuelle de microsatellites très informatifs et l'avènement d'une carte dense du génome humain permet d'augmenter les chances de détecter une liaison [5]. Une étude récente a montré une liaison forte avec des marqueurs en 6p22-pter [6], et ce résultat demande confirmation**.

Des analyses de liaison venant de deux groupes ont également suggéré la présence d'un locus intervenant dans l'origine de la schizophrénie dans la région chromosomique

\footnotetext{
* Phénocopie : état d'un sujet dont le phénotype évoque celui d'une affection d'origine génétique sans que celle-ci puisse être mise en évidence. ** Voir note ajoutée aux épreuves, p. 1731.
}

22q12-q13.1 [7, 8]. Cependant, l'analyse d'un plus grand nombre de familles (256) n'a pas détecté de liaison significative, suggérant que seul un faible nombre de familles serait lié à ce locus [9]. L'hétérogénéité génétique de la maladie apparaît certaine [10] et les résultats de différentes équipes sur la potentialité d'une liaison au chromosome $22 \mathrm{q}$ sont actuellement discordants [11-13]. Une étude récente multicentrique [14] portant sur 695 schizophrènes et 18 patients atteints de syndrome vélocardio-facial (VCF) (dont quatre présentant les critères de schizophrénie du DSM-III-R) fait estimer à $2 \%$ la fréquence des patients schizophrènes porteurs d'une délétion 22q11 par hybridation in situ en fluorescence (FISH) avec la sonde Scl1.1, et conclure que l'hémizygotie 22q11 est un facteur prédisposant à la schizophrénie. L'étendue des délétions chez cinq patients de cette étude a été analysée à l'aide de microsatellites. La région minimale critique des patients schizophrènes est bornée entre les loci D22S264 et D22S427 (environ $2 \mathrm{Mb}$ ), et contient le gène candidat de la catéchol-O-méthyltransférase (COMT). Cependant, des délétions distales à D22S264 ont été retrouvées chez des patients atteints de syndrome VCF sans schizophrénie, ce qui suggère qu'il n'existe pas de corrélation au niveau de la borne distale avec le phénotype psychiatrique ajouté. Dans l'état actuel des choses, les bornes proximales de délétion chez les patients avec et sans schizophrénie ne peuvent être distinguées, et des marqueurs supplémentaires devront être testés pour rechercher d'éventuelles différences dans l'étendue des délétions. Ces résultats sont 
schématisés sur la figure 1. Les patients porteurs d'une délétion présentent tous des caractères dysmorphiques faciaux, évocateurs de syndrome VCF. Il est connu que plus de $10 \%$ des patients VCF développent des troubles psychiatriques, le plus souvent une schizophrénie chronique de type paranoïde [15]. Les patients schizophrènes porteurs d'une délétion interstitielle 22q11 présentent des critères mineurs de phénotype VCF qui peuvent rester méconnus en l'absence d'évaluation dysmorphologique [16]. Des anomalies morphologiques mineures (replis épicanthiques*, anomalies des oreilles, palais ogival) sont d'ailleurs retrouvées plus souvent chez les schizophrènes que dans une population témoin [17], ce qui suggère une possible implication d'un gène du développement chez certains patients. Un deuxième locus de prédisposition pourrait cependant exister sur le bras long du chromosome $22(22 q 12)$ [18], comme semblent l'indiquer les résultats des analyses de liaison $[7,8,12]$, et aucune région du chromosome $22 q$ ne peut être définitivement exclue actuellement [19].

L'acronyme CATCH 22 regroupe certains signes communs à différents syndromes associés à une microdélétion $22 q 11$ identifiable par FISH [20]. Cette microdélétion du chromosome $22 q 11$ est fréquente $(1 / 4000$ naissances, $0,025 \%$ ) et retrouvée dans $90 \%$ à $100 \%$ des cas de syndrome de DiGeorge $\left(\mathrm{m} / \mathrm{s} n^{\circ} 6\right.$, vol. $7, p$. $618 ; n^{\circ} 2$, vol. 8, p. 182), $70 \%$ à $80 \%$ des cas de syndrome VCF [21], des formes familiales de cardiopathies cono-truncales [22], le syndrome cono-trunco-facial [23], et de rares observations d'association CHARGE (AC) $(m / s ~ n \circ 8$, vol. 11, p. 1183). Les formes typiques de cette dernière anomalie polymalformative ne s'avèrent pas liées à une délétion du chromosome 22. L'asymétrie faciale aux pleurs qui est un critère majeur de l'AC définit, en association à une anomalie cardiaque, le syndrome cardio-facial de Cayler. La recherche

* Replis cutanés verticaux à concavité externe recouvrant plus ou moins, en le masquant, l'angle palpébral interne.

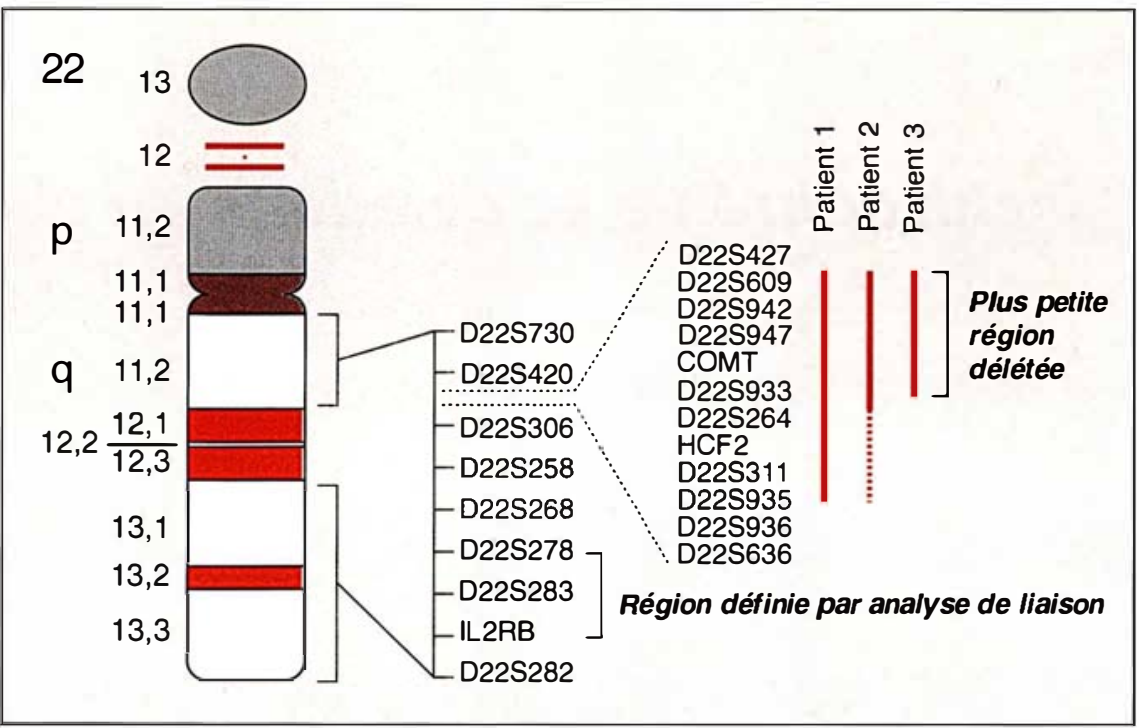

Figure 1. Idéogramme du chromosome 22 indiquant la région distale candidate pour un locus de prédisposition à la schizophrénie, et la région proximale définie par l'analyse des délétions. Les patients 1, 2 et 3 portent les types de délétions observées dans [14]. Les délétions sont schématisées par des traits verticaux. L'étendue exacte de la délétion du patient 2 n'est pas connue.

d'une microdélétion $22 q 11$ dans ce dernier syndrome a été suggérée [24] et récemment retrouvée chez cinq patients [25]. D'autres phénotypes plus inhabituels ont été décrits en association à la délétion $22 \mathrm{q} 11$, comme des anomalies urologiques $\left(\mathrm{m} / \mathrm{s} n^{\circ} 8\right.$, vol. 11, p. 1183) (20\%), des malformations laryngées ou des anomalies des extrémités (pouce triphalangien, mains botes radiales, polydactylie pré-axiale) [26]. Certains critères mineurs dysmorphiques de la face apparaissent comme de bons marqueurs cliniques pour rechercher l'anomalie moléculaire du chromosome 22q11, comme l'aspect du nez (pseudo-bulbaire et long avec une hypertrophie des os propres du nez, une racine proéminente, et une hypoplasie des ailes du nez) et l'aspect des oreilles (asymétriques, larges, décollées, dysplasiques avec hélix déficient et diamètre antéropostérieur augmenté, et volontiers bas implantées en rotation postérieu- re). Les autres critères mineurs faciaux inconstamment retrouvés sont: hypertélorisme* ou télécanthus, microstomie, lèvres fines, et asymétrie faciale aux pleurs.

Le phénotype clinique du CATCH22 apparaît polymorphe. L'expression clinique est variable entre les familles et pour les divers syndromes, et également à l'intérieur des familles avec des phénotypes distincts dans la même famille [27]. Les différences inter-familiales ne semblent pas liées à des délétions de taille différente selon les familles ni à une anomalie de gènes contigus. Il semble très probable que des modifications épigénétiques interviennent, et des différences ethniques pourraient exister également. Les différences intra-familiales (qui semblent très fréquentes) peuvent avoir de nombreuses origines: mosaïque

* Élargissement anormal de l'espace interoculaire. $\mathrm{m} / \mathrm{s} n^{\circ} 12$, vol. 11 , décembre 95 
parentale (avec absence de mosaïcisme chez l'enfant sévèrement affecté), mutation instable de l'ADN par expansion trinucléotidique (avec accentuation de la répétition au fil des générations), empreinte parentale (différence d'expression génique selon la transmission paternelle ou maternelle de la délétion [28]), complémentation génétique, polyallélisme, effet lié à l'environnement, gènes modificateurs à des loci différents... [29]. Un phénomène d'empreinte génétique semble actuellement écarté [21]. L'hypothèse actuelle favorise l'idée d'un gène majeur intervenant dans la genèse des $3^{\mathrm{e}}$ et $4^{\mathrm{c}}$ arcs branchiaux. La délétion retrouvée est le plus souvent étendue, de l'ordre de $2000 \mathrm{~kb}$. La zone minimale critique est actuellement de l'ordre de $300 \mathrm{~kb}$. L'hypothèse pathogénique actuelle favorise plutôt l'idée d'un gène unique que de gènes contigus. Des gènes candidats sont en cours d'étude comme le gène COMT, un gène régulateur de la transcription des histones (HIRA) [30] qui est analogue à TUPLE 1 (tup-like enhancer of split gene 1) [31], ou le gène DGCR2 (DiGeorge syndrome critical region 2) [32].

Une origine préférentielle de transmission de la délétion d'origine maternelle est parfois retrouvée [33, 34]. Le phénotype des mères transmettrices associe des critères faciaux constants à une présentation volontiers psychiatrique [35]. Le CATCH22 est une affection dysmorphogénétique de transmission autosomique dominante et définit un groupe d'anomalies du développement relativement fréquentes dont l'expressivité phénotypique est extrêmement variable. Un diagnostic prénatal par recherche de la délétion 22 q11 par FISH est possible dans les formes familiales [36], mais le conseil génétique doit rester prudent en raison de la variabilité clinique.

La recherche d'une hémizygotie 22q11 chez les patients schizophrènes apparaît d'intérêt en présence de critères dysmorphiques évocateurs de syndrome VCF, et on ne peut pas exclure que le même gène soit responsable des différents syndromes dysmorphogénétiques et des troubles psychiatriques observés en association à la microdélétion $22 q 11$. La région chro- mosomique 22q11-q13 apparaît actuellement comme une région candidate de prédisposition à la schizophrénie, mais l'hétérogénéité génétique de la maladie implique de rechercher d'autres loci. Devant les difficultés posées par les analyses de liaison, d'autres stratégies ont été mises en œuvre: études non paramétriques, études d'association, recherche de mutations dans des gènes candidats (récepteurs de la dopamine essentiellement). Aucun résultat significatif n'a pu être obtenu par ces différentes approches jusqu'à présent. Cependant, l'approche par gène candidat pourrait inclure des candidats aussi divers que les gènes intervenant dans la médiation et le métabolisme des neurotransmetteurs, ou des gènes participant au développement et à la migration neuronale. Une autre approche est celle du clonage positionnel par recherche d'association de la maladie à des anomalies cytogénétiques. Certaines translocations chromosomiques équilibrées ont été décrites [37, 38] avec des points de cassure en lq, 6q et 11q. Un programme visant à cloner le point de cassure de la translocation $t$ $(1 ; 11)(q 42.3 ; q 14.3)$ est en cours [39]. Enfin, un phénomène d'anticipation paraît intervenir dans certaines familles de schizophrènes [40]. Ce phénomène trouve son explication dans certaines maladies (chorée de Huntington, dystrophie myotonique de Steinert par exemple) par l'amplification de trinucléotides au sein des gènes responsables. Grâce à la technique RED (repeat expansion detection) [41] qui permet de rechercher des expansions trinucléotidiques de façon globale dans le génome, O'Donnovan et al. [42] ont rapporté que les patients schizophrènes présentent une plus grande tendance à l'amplification de triplets CAG qu'un groupe témoin. Ce résultat suggère que des mutations instables de l'ADN pourraient être impliquées dans le déterminisme des maladies psychiatriques. Cette dernière piste demande confirmation, et des gènes contenant une expansion trinucléotidique devraient alors être recherchés dans les régions chromosomiques candidates.

L'identification de gènes de prédisposition à la schizophrénie reste l'un des défis actuels de la génétique médicale

\section{Didier Lacombe}

Service de pédiatrie et génétique médicale, hôpital Pellegrin-Enfants, université de Bordeaux II, place Amélie-Raba-Léon, 33076 Bordeaux Cedex, France.

\section{Benoît Arveiler}

Laboratoire de pathologie moléculaire et de thérapie génique, université de Bordeaux II, 33076 Bordeaux, France.

\section{RÉFÉRENCES}

1. Kendler KS, Diehl SR. The genetics of schizophrenia: a current, genetic-epidemiologic perspective. Schizo Bull 1993; 19: 26185.

2. American Psychiatric Association. DSM III-R: Diagnostic and statistical manual of men tal disorders. 3rd ed., revised. Washington, DC: The Association, 1987.

3. Pauls DL. Behavioural disorders: lessons in linkage. Nature Genet 1993; $3: 4-5$.

4. Cloninger CR. Turning point in the design of linkage studies of schizophrenia. Am JMed Genet 1994; 54: 83-92.

5. Weissenbach J. Le génome humain entre médecine et science. médecine/sciences 1995 ; $11: 317-23$

6. Wang S, Sun CE, Walczak CA, Ziegle JS, Kipps BR, Goldin LR, Diehl SR. Evidence for a susceptibility locus for schizophrenia on chromosome 6pter-p22. Nature Genet $1995 ; 10: 41-6$.

7. Pulver AE, Karayiorgou M, Wolyniec PS, Lasseter VK, Kasch L, Nestadt G, Antonarakis S, Housman D, Kazazian HH, Meyers D, Ott J, Lamacz M, Liang KY, Hanfelt J, Ullrich G, DeMarchi N, Ramu E, McHugh PR, Adler L, Thomas M, Carpenter WT, Manschreck T, Gordon CT, Kimberland $M$, Babb R, Puck J, Childs B. Sequential strategy to identify a susceptibility gene for schizophrenia: report of potential linkage on chromosome 22q12-q13: Part 1. Am J Med Genet 1994; 54: 36-43 
8. Coon H, Holik J, Hoff M, Reimherr F, Wender P, Myles-Worsley $M$, Waldo $M$, Freedman R, Byerley W. Analysis of chromosome 22 markers in nine schizophrenia pedigrees. Am J Med Genet 1994; 54 : 72-9.

9. Pulver AE, Karayiorgou M, Lasseter VK, Wolyniec P, Kash L, Antonarakis S, Housman D, Kazazian HH, Meyers D, Nestadt G Ott J, Liang KY, Lamacz M, Thomas $M$, Childs B, Diehl SR, Wang S, Murphy B, Sun C, O'Neill FA, Nie L, Sham P, Burke J, Duke BW, Duke F, Kipps BR, Bray J, Hunt W Shinkwin R, Nuallain MN, Su Y, MacLean CJ, Walsh D, Kendler KS, Gill M, Vallada $H$ Mant R, Asherson P, Collier D, Parfitt E, Roberts E, Nanko S, Walsh C, Daniels J, Murray $R$, MacGuffin $P$, Owen $M$, Laurent $C$, Dumas JB, d'Armato T, Jay M, Martinez $M$ Campion D, Mallet J. Follow-up of a report of a potential linkage for schizophrenia on chromosome 22q12-q13.1 : Part 2. AmJ Med Genet $1994 ; 54: 44-50$.

10. Coon $H$, Jensen $S$, Holik J, Hoff $M$ Myles-Worsley M, Reimherr F, Wender P, Waldo M, Freedman R, Leppert M, Byerley W. Genomic scan for genes predisposing to schizophrenia. Am J Med Genet 1994; $54: 59$ 71 .

11. Polymeropoulos $\mathrm{MH}$, Coon $\mathrm{H}$, Byerley W, Gershon ES, Goldin L, Crow TJ, Rubenstein J, Hoff M, Holik J, Smith AM, Shields G, Bass NJ, Poulter M, Lofthouse R, Vita A Morganti C, Merril CR, Delisi LE. Search for a schizophrenia susceptibility locus on human chromosome 22. Am J Med Genet $1994 ; 54: 93-9$

12. Vallada HP, Gill M, Sham P, Lim LCC Nanko S, Asherson P, Murray RM, McGuffin P, Owen M, Collier D. Linkage studies on chromosome 22 in familial schizophrenia. Am J Med Genet 1995; $60: 139-46$.

13. Kalsi G, Brynjolfsson J, Butler R, Shenington $R$, Curtis $D$, Sigmundsson $T$, Read T, Murphy P, Sharma T, Petursson H, Gurling HMD. Linkage analysis of chromosome 22q12-13 in a United Kingdom/Iceland sample of 23 multiplex schizophrenia families. Am J Med Genet 1995; 60 : 298-301.

14. Karayiorgou M, Morris MA, Morrow B, Shprintzen RJ, Goldberg R, Borrow J, Gos A, Nestadt G, Wolyniec PS, Lasseter VK, Eisen $\mathrm{H}$, Childs B, Kazazian HH, Kucherlapat $\mathrm{R}$, Antonarakis SE, Pulver AE, Housman DE. Schizophrenia susceptibility associated with interstitial deletions of chromosome 22q11. Proc Natl Acad Sci USA 1995; 92: $7612-6$.

15. Shprintzen RJ, Goldberg R, Golding-Kushner KJ, Marion RW. Late-onset psychosis in the velo-cardio-facial syndrome. Am J Med Genet 1992; 42 : 141-2.

16. Lindsay EA, Morris MA, Gos A, Nestadt G, Wolyniec PS, Lasseter WK, Shprintzen R, Antonarakis SE, Baldini A, Pulver AE. Schizophrenia and chromosomal deletions within 22q11.2 (letter). Am J Hum Genet 1995; 56: 1502-3.

17. Lane A, Cassidy B, Murphy P, Sheppard $\mathrm{N}$, Horgan $\mathrm{R}$, Keenan J, Waddington JL, Larkin C, O'Callaghan E. Minor physical anomalies in schizophrenia. Irish J Med Sci
18. Propping P, Nöthern MM. Schizophrenia: genetic tools for unraveling the nature of a complexe disorder (commentary). Proc Natl Acad Sci USA 1995; 92: 7607-8.

19. Lasseter VK, Pulver AE, Wolyniec PS, Nestadt G, Meyers D, Karayiorgou M, Housman D, Antonarakis S, Kazazian H, Kasch L, Babb R, Kimberland M, Childs B. Follow-up report of potential linkage for schizophrenia on chromosome 22q: part 3 (letter) Am J Med Genet 1995; 60 : 172-3.

20. Wilson DI, Burn J, Scambler PJ, Goodship J. DiGeorge syndrome: part of CATCH 22. JMed Genet 1993; 30: 852-6.

21. Morrow B, Goldberg R, Carlson C, Das Gupta R, Sirotkin H, Collins J, Dunham I, O'Donnell H, Scambler P, Shprintzen R Kucherlapati R. Molecular definition of the 22q11 deletions in velo-cardio-facial syndrome. Am J Hum Genet 1995; 56: 1391-403.

22. Wilson DI, Goodship JA, Burn J, Cross IE, Scambler PJ. Deletions within chromosome 22q11 in familial congenital heart disease. Lancet 1992; 340: 573-5.

23. Matsuoka R, Takao A, Kimura M, Imamura SI, Kondo C, Joh-o K, lkeda K, Nishibatake $M$, Ando $M$, Momma $K$. Confirmation that the conotruncal anomaly face sundrome is associated with a deletion within 22q11.2. Am J Med Genet 1994; 53 : 285-

24. Lacombe D. Facial palsy and cranial nerve abnormalities in CHARGE association. AmJ Med Genet 1994; 49:351-2.

25. Giannotti A, Digilio MC, Marino B, Mingarelli R, Dallapiccola B. Cayler cardiofacial syndrome and del 22q11: part of the CATCH 22 phenotype. Am J Med Genet 1994; 53 : 303-4.

26. Cormier-Daire V, Iserin L, Théophile D, Sidi D, Vervel C, Padovani JP, Vekemans M, Munnich A, Lyonnet S. Upper limb malformations in DiGeorge syndrome. Am J Med Genet 1995; 56: 39-41.

27. Stevens CA, Carey JC, Shigeoka AO. DiGeorge anomaly and velocardiofacial syndrome. Pediatrics 1990; 85: 526-30.

28. Babinet C. L'empreinte génomique parentale. médecine/sciences 1992; 8: 65-70.

29. Hall JG. CATCH 22 (éditorial). J Med Genet 1993; 30: 801-2.

30. Lamour V, Lécluse Y, Desmaze C, Spector M, Bodescot M, Aurias A, Osley MA, Lipinski $M$. A human homolog of the $S$. cerevisiae HIR1 and HIR2 transcriptional repressors cloned from the DiGeorge syndrome critical region. Hum Mol Genet 1995 ; 4: 791-9.

31. Halford S, Wadey R, Roberts G, Daw SCM, Whiting JA, O'Donnel H, Dunham I, Bentley D, Lindsay E, Baldini A, Francis F, Lehrach $\mathrm{H}$, Williamson R, Wilson DI, Goodship J, Cross I, Burn J, Scambler PJ. Isolation of a putative transcriptional regulator from the region of $22 \mathrm{qll}$ deleted in DiGeorge syndrome, Sphrintzen syndrome and familial congenital heart disease. Hum Mol Genet 1993 ; 2: 2099-107.
32. Demczuk S, Aledo R, Zucman J, Delattre $O$, Desmaze C, Dauphinot L, Jalbert P, Rouleau GA, Thomas G, Aurias A. Cloning of a balanced translocation breakpoint in the DiGeorge syndrome critical region and isolation of a novel potential adhesion recep tor gene in its vinicity. Hum Mol Genet 1995; 4: 551-8.

33. Seaver LH, Pierpont JW, Erickson RP, Donnerstein RL, Cassidy SB. Pulmonary atresia associated with maternal $22 \mathrm{q} 11.2 \mathrm{de}$ letion: possible parent of origin effect in the conotruncal anomaly face syndrome. $J$ Med Genet 1994; 31 : 830-4.

34. Demczuk S, Aurias A. DiGeorge syndrome and related syndromes associated with 22q11.2 deletions. A review. Ann Genet $1995 ; 38$ : 59-76.

35. Philip N, Levy A, Le Merrer M, Lacombe D. Natural history of CATCH 22: lessons from the study of carrier patients. 6th Manchester Birth Defects Conference. Holly Royde (UK), 1994.

36. Driscoll DA, Salvin J, Sellinger B, Budarf ML, MacDonald-McGinn DM, Zackai EH, Emanuel BS. Prevalence of 22q11 microdeletions in DiGeorge and velocardiofacial syndromes: implications for genetic counselling and prenatal diagnosis. $J$ Med Genet $1993 ; 30: 813-7$.

37. Holland T, Gosden CM. A balanced chromosomal translocation partially co-segregating with psychotic illness in a family. Psychiatry Res 1990; 32: 1-8.

38. St Clair DM, Blackwood DHR, Muir WJ, Carothers A, Walker M, Spowart G, Gosden CM, Evans HJ. Association within a family of a balanced autosomal translocation with major mental illness. Lancet 1990; 336: 13-6.

39. Evans KL, Brown J, Shibasaki Y, Devon RS, He L, Arveiler B, Christie S, Maule JC, Baillie D, Slorach EM, Anderson SM, Gosden JR, Petit J, Weith A, Gosden CM, Blacwood DHR, St-Clair DM, Muir WJ, Brookes AJ, Porteous DJ.A contiguous clone map over $3 \mathrm{Mb}$ on the long arm of chromosome 11 across a balanced translocation associated with schizophrenia. Genomics 1995; 28 : $420-8$.

40. Bassett AS, Honer WG. Evidence for anticipation in schizophrenia. Am J Hum Genet $1994 ; 54: 864-70$.

41. Schalling M, Hudson TJ, Buetow $\mathrm{KH}$; Housman DE. Direct detection of novel expanded trinucleotide repeats in the human genome. Nature Genet 1993; 4: 135-9.

42. O'Donovan MC, Guy C, Craddock N, Murphy KC, Cardno AG, Jones LA, Owen MJ, Mc Guffin P. Expanded CAG repeats in schizophrenia and bipolar disorder. Nature Genet 1995; 10 : 380-1.

43. Shprintzen RJ, Goldberg RB, Lewin ML, Sidoti EJ, Berkman MD, Argamaso RV Young D. A new syndrome involving cleft palate, cardiac anomalies, typical facies, and learning disabilities: velo-cardio-facial syndrome. Cleft Palate J 1978; $15: 56-62$. 


\section{RÉFÉRENCES}

44. Mitnick RJ, Bello JA, Shprintzen RJ Brain anomalies in velo-cardio-facial syndrome. Am J Med Genet 1994 ; 54 : 100-6.

45. Straub RE, MacLean CJ, O'Neill FA, Burke J, Murphy B, Duke F, Shinkwin R Webb BT, Zhang J, Walsh D, Kendler KS, A potential vulnerability locus for shizophrenia on chromosome 6p24-22: evidence for genetic heterogeneity. Nature Genet 1995 11: $287-93$.

46. Moises HW, Yang L, Kristbjarnarson $\mathrm{H}$ et al. (28 auteurs). An international two-stage genome-wide search for shizophrenia susceptibility genes. Nature Genet $1955 ; 11$. 321-7.

47. Mowry BJ, Nancarrow DJ, Lennon DP, et al. (13 auteurs). Schizoprenia susceptibility and chromosome 6p24-22. Nature Genet $1995 ; 11: 233-4$.

48. Schwab SG, Albus M, Hallmayer J, et al. (12 auteurs). Evaluation of a susceptibility gene for schizophrenia on chromosome $6 \mathrm{p}$ by multipoint affected sib-pair linkage analysis. Nature Genet 1995; 11 : 325-7.

\section{Note ajoutée aux épreuves}

Cette localisation en 6p24-22 est confirmée dans une étude de pedigrees irlandais plus importante que celle rapportée dans la référence 6 [45] avec un lod score de 3,51 avec un marqueur particulier. Cette même localisation apparaît dans une étude internationale multicentrique, associée à de possibles loci sur les chromosomes 9 et 20 [46]; en particulier, le locus en $6 p$ pourrait jouer un rôle dans les pedigrees chinois. En revanche, il ne ressort pas d'une étude de pedigrees australiens et américains [47] et européens [46]; cependant, une quatrième étude portant sur des familles allemandes et juives sépharades d'Israël est en faveur de ce locus en $6 p$ [48]. Ces récents résultats confirment les incertitudes sur la génétique de la schizophrénie qui est, au mieux, hétérogène et à pénétrance faible.

\section{* GLOSSAIRE}

Association CHARGE : association polymalformative définie par un acronyme:

C - Coloboma (colobome oculaire)

$H$ - Heart disease (cardiopathie congénitale)

A - Atresia choanae (atrésie des choanes)

$R$ - Retarded growth (retard de croissance)

Retarded development (retard psychomoteur)

$G$ - Genital hypoplasia (anomalies génitales)

E - Ear anomalies and/or deafness (anomalies des oreilles et/ou surdité)

CATCH22 : spectre acronymique regroupant des symptômes les plus fréquemment retrouvés avec la microdélétion $22 q 11$ :

$C$ - Cardiac defects (malformations cardiaques)

A - Abnormal facies (dysmorphie faciale)

$T$ - Thymic hypoplasia (hypoplasie thymique)

$C$ - Cleft palate (fente palatine)

$H$ - Hypocalcemia (hypocalcémie)

22 - Délétion 22q11.

Syndrome cardio-facial de Cayler : association d'une malformation cardiaque et d'une asymétrie faciale aux pleurs due à une amyoplasie (aplasie ou hypoplasie) $d u$ muscle triangulaire des lèrres.

Syndrome cono-trunco-facial : syndrome décrit au Japon et associant cardiopathie congénitale, anomalies faciales (hypertélorisme, fentes palpébrales courtes, aplatissement de la racine du nez, anomalies mineures des oreilles), et retard mental modéré.

Syndrome de DiGeorge : syndrome caractérisé par l'association d'une aplasie ou hypoplasie thymique, d'une hypocalcémie par absence ou hypoplasie des parathyroïdes, d'une cardiopathie congénitale de type cono-truncale, et d'une dysmorphie faciale discrète avec microstomie, fentes palpébrales étroites, hypertrophie des os propres $d u$ nez.

Syndrome vélo-cardio-facial (VCF) : syndrome décrit par Shprintzen [43], associant une fente palatine, une insuffisance vélaire (90\%), une malformation cardiaque (80\%), des difficultés d'apprentissage (95\%), un retard mental (40\%), des anomalies cérébrales [44], des troubles psychiatriques (15\%), une dysmorphie faciale caractéristique (nez pseudo-bulbaire, dysplasie des oreilles), et un aspect long et effilé des doigts.

\section{TIRÉS À PART}

D. Lacombe 